\title{
Instituto Mexicano del Petróleo
}

\author{
Jaime Sánchez Valente ${ }^{1}$ y José Antonio Toledo Antonio ${ }^{2}$
}

RESUMEN: En el mundo actual es indudable que el uso de la nanotecnología es indispensable, para la manipulación de los materiales desde sus unidades básicas de construcción, con el fin de controlar las distintas propiedades fisicoquímicas de los mismos. Por tal motivo, en el presente trabajo se expone el empleo de la nanotecnología en el Instituto Mexicano del Petróleo (IMP), como una herramienta para el diseño y desarrollo de nuevos materiales, así como la infraestructura con la que cuenta el IMP para su caracterización, escalamiento y evaluación a nivel micro y piloto.

PALABRAS CLAVE: Nanotecnología, catalizadores, desarrollo tecnológico, petróleo.

ABSTRACT: It is indubitable that in our modern world, nanotechnology is fundamental for the manipulation of materials from their basic building blocks, so that their physicochemical properties may be controlled. For this reason, herein is presented the use of nanotechnology at the Mexican Petroleum Institute (IMP) as a tool for the design and development of novel materials. Also the infrastructure that IMP has for the characterization, scaling-up, and evaluation, at micro and pilot level, of nanomaterials.

KEYWORDS: Nanotechnology, catalysts, technological development, oil.

El Instituto Mexicano del Petróleo (IMP), creado el 23 de agosto de 1965, es el centro de investigación de México dedicado al área petrolera, cuyos objetivos principales son la investigación y desarrollo tecnológico, así como la formación de recursos humanos de alto nivel. Proporciona, también, servicios técnicos y capacitación a la industria del petróleo, además de la comercialización de los desarrollos tecnológicos, ya sea por cuenta propia o bajo la suscripción de alianzas con instituciones o empresas privadas.

Actualmente, debido a la apertura del mercado petrolero en el país, el IMP ha sido transformado en un instituto nacional. Adaptándose en consecuencia a los nuevos objetivos y grandes retos de la industria petrolera, mediante renovados esfuerzos basados en la investigación y desarrollo tecnológico, con el enfoque a mejorar la eficiencia, productividad y crecimiento del sector de hidrocarburos.

Recibido: 27 de junio de 2016. Aceptado: 28 de octubre de 2016.

1 Gerente de desarrollo de materiales y productos químicos, Instituto Mexicano del Petróleo, Edificio 23, Oficina 204, Eje Central L. Cardenas \# 152, Col. Sn Bartolo Atepehuacan, Del. G. A. Madero, 07730 Ciudad de Mexico. Correspondencia: (jsanchez@imp.mx), Tel. (52) 55 91758413.

2 Líder de especialidad en el Instituto Mexicano del Petróleo, Edificio 23, Oficina 221, Eje Central L. Cardenas \# 152, Col. Sn Bartolo Atepehuacan, Del. G. A. Madero, 07730 Ciudad de Mexico. Correspondencia: (jtoledo@imp.mx), Tel. (52) 5591758434. 
FIGURA 1. Entrada principal y torre ejecutiva del IMP.

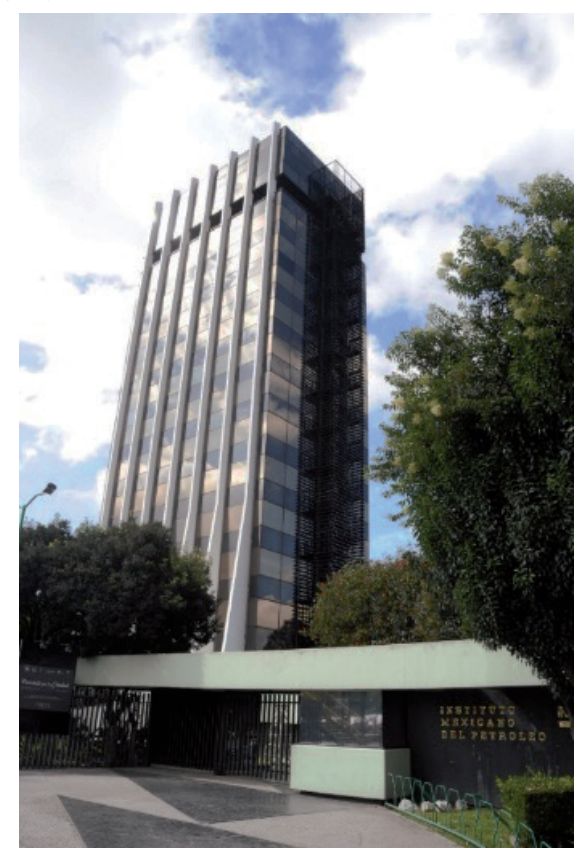

Como centro público de investigación, el Instituto Mexicano del Petróleo tiene la misión de transformar el conocimiento en tecnología y servicios de valor para la industria petrolera; y la visión de ser un centro público de investigación de clase mundial con personal reconocido, con tecnologías y servicios que contribuyan al desarrollo de la industria petrolera.

Considerando que la manera de crear valor económico, a partir de los hidrocarburos, es a través de procesos de transformación química, y sabiendo que alrededor del $90 \%$ de tales procesos emplean algún tipo de catalizador, es claro que la catálisis tiene un papel de suma importancia; principalmente la catálisis heterogénea. En este sentido, desde sus orígenes, el IMP ha desarrollado metodologías de síntesis de catalizadores heterogéneos, buscando siempre controlar las estructuras cristalinas, es decir, los arreglos atómicos que constituyen el compuesto, así como la superficie expuesta del mismo y eventualmente la dispersión de los componentes activos, en los catalizadores heterogéneos que se emplean en la industria petrolera.

En 1836, Berzelius acuña el término "catalizador" para referirse a una sustancia que desempeña un rol central en una reacción química, sin sufrir una transformación en sí misma. En concreto, su función principal es la de disminuir las barreras energéticas y orientar selectivamente la formación de los productos deseados. De tal forma, la catálisis heterogénea es un campo interdisciplinario de la ciencia y la tecnología, que hace uso de la química, la 
FIGURA 2. Vista aérea de las instalaciones del IMP sede.

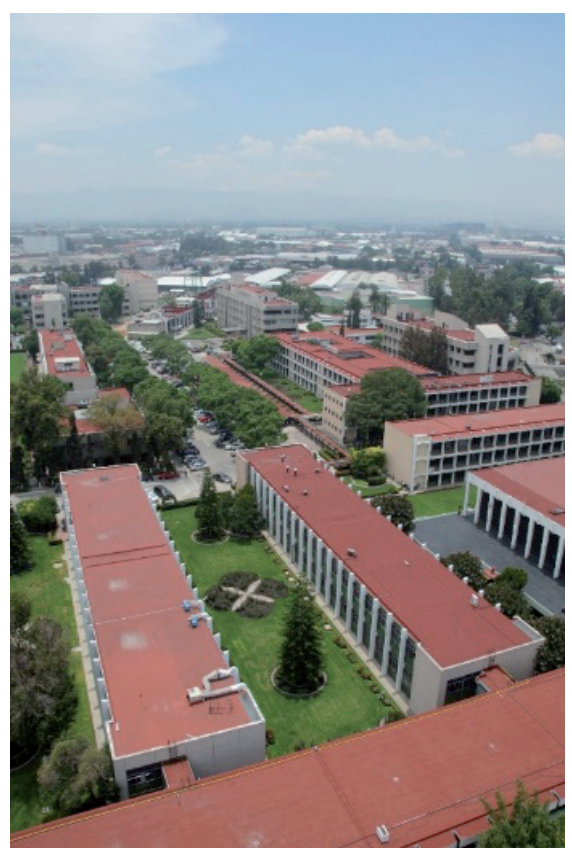

ingeniería, la física del estado sólido, la biología y la rama de la ciencia conocida hoy en día como nanotecnología, entre otras disciplinas.

Cabe hacer mención de que los catalizadores empleados en la industria siempre se han manejado a escala nanométrica. Sin embargo, no fue sino hasta los años 80's, debido a los avances de distintas tecnologías, en particular la relacionada con los microscopios electrónicos, que ha sido posible manipular y observar de manera precisa los materiales a escala nanométrica, lográndose así importantes avances en el diseño de los catalizadores más eficientes, debido a que los materiales nanoestructurados ( $1 \mathrm{~nm}=10-9$ metros) exhiben propiedades electrónicas únicas, reflejándose la mayoría de las veces en su reactividad y selectividad química, permitiendo sorprendentes e impredecibles ventajas en sus diversas aplicaciones.

En catálisis, los nanomateriales catalizan reacciones químicas con más altas conversiones y selectividades, logrando altos rendimientos hacia el o los productos deseados y con mayor eficiencia energética, comparado con los catalizadores convencionales, lo cual se traduce en ventajas tecnológicas y económicas considerables, por ejemplo, lograr disminuir el contenido de metal noble en catalizadores para procesos de hidrogenación y reformación, desde concentraciones de $3.0 \%$ peso hasta $<0.3 \%$ peso de metal.

La nanotecnología es una rama de la ciencia dedicada a la manipulación de materiales desde sus unidades básicas de construcción: los átomos, las moléculas, y el estudio de las partículas de tamaños del orden desde 1 a $100 \mathrm{~nm}$; 
FIGURA 3. (a) Materiales con morfología nanotubular y dimensiones nanométricas, (b) metales soportados con dimensiones $<5.0 \mathrm{~nm}$, (c) metales soportados con dimensiones < $1.0 \mathrm{~nm}, \mathrm{y}$, (d) estructura cristalina de una nanopartícula metálica soportada.

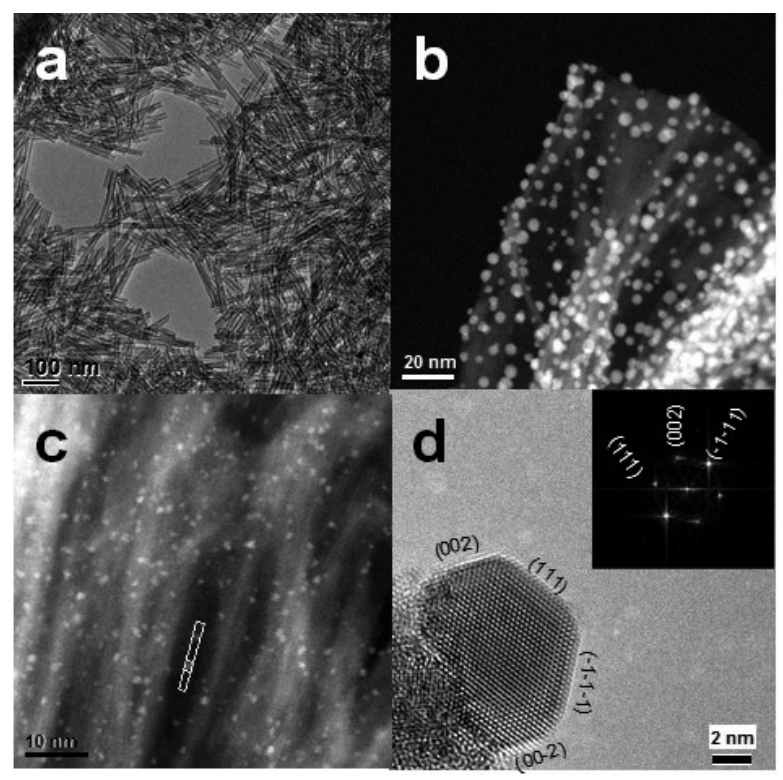

es decir, desde una millonésima hasta una diezmilésima parte de un milímetro. A esta escala, la materia tiene propiedades especiales que, por mencionar alguna, le ayudan a reaccionar más fácilmente con otras moléculas.

En el Instituto Mexicano del Petróleo se estudian la manipulación y control de distintos materiales, usando las herramientas disponibles con las que cuenta la institución. En este sentido, el control de nanopartículas ha sido de gran importancia, pues éstas tienen a menudo propiedades catalíticas importantes y distintas a cuando esos mismos compuestos se presentan en forma de partículas de mayor tamaño.

La nanotecnología hoy en día se define como la habilidad para construir artefactos mediante la manipulación de átomos o moléculas a través de estrategias denominadas "de abajo a arriba" utilizando técnicas y herramientas desarrolladas para hacer productos de más alto rendimiento.

\section{Líneas de investigación en nanotecnología}

En las muy diversas reacciones químicas de interés para el IMP (refinación de petróleo, producción de hidrocarburos y productos petroquímicos, purificación de combustibles, entre muchas otras) intervienen comúnmente diversos materiales y catalizadores. Una manera de mejorar el desempeño de tales compuestos es mediante la manipulación de los arreglos atómicos a escala nanométrica durante su formación. En el IMP se estudia la nanotecnología 
aplicada al desarrollo de las distintas líneas de investigación; se destacan, entre otras, las siguientes:

- Producción de combustibles limpios.

- Oxidación parcial de parafinas ligeras.

- Aprovechamiento del gas natural (transformación de metano y etano a productos químicos y petroquímicos de mayor valor agregado).

- Transformación de $\mathrm{CO}_{2}$ a productos químicos y petroquímicos.

- Producción de hidrógeno.

- Películas delgadas.

- Biomateriales.

- Biocombustibles.

- Productos químicos.

- Tecnologías para el desarrollo y explotación de yacimientos convencionales y no convencionales.

- Mejoramiento de hidrocarburos no convencionales.

- Fotocatálisis de contaminantes recalcitrantes en medio acuoso.

\section{Nanotecnología computacional en el IMP}

La nanotecnología computacional está basada en la modelación molecular y la simulación a multiescala, lo cual permite el desarrollo de modelos que representan los fenómenos fisicoquímicos, considerando las propiedades electrónicas moleculares y termodinámicas del compuesto en estudio. De

FIGURA 4. Análisis por microscopía electrónica de películas delgadas, preparadas por el método solgel, de hidróxidos dobles laminares.

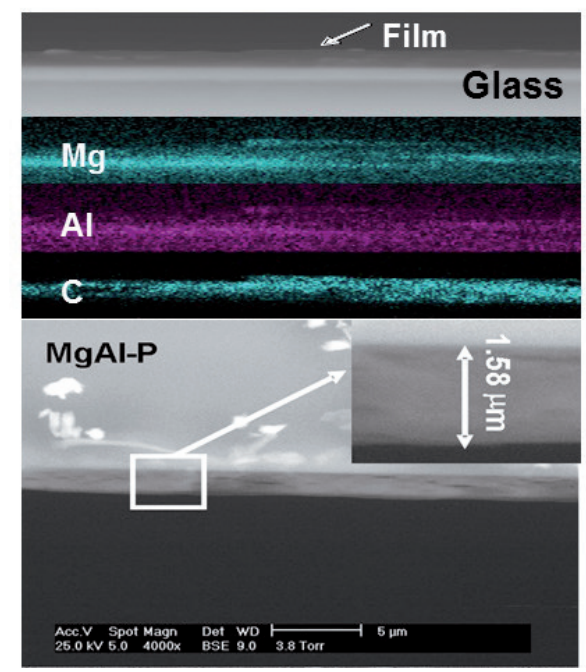


este modo se abarcan los dominios microscópico, mesoscópico, y nanométrico. Los modelos así desarrollados permiten la solución de problemas no sólo científicos, sino incluso tecnológicos, creando la base para la generación y establecimiento de la relación teoría-industria. Las aplicaciones de la nanotecnología requieren un conocimiento profundo de los aspectos teóricos de los materiales y dispositivos a escala nanométrica. Por tal motivo, diversas áreas, en específico la química computacional con la gran diversidad de aproximaciones a multiescala, permiten el estudio de los materiales.

La química computacional es esencial para la integración y comprensión entre las escalas atómica y molecular con el mundo micro, meso y macroscópico. Los métodos empleados en esta área han avanzado considerablemente, dentro de éstos se encuentran: la teoría de los funcionales de la densidad (DFT), la mecánica molecular, los métodos de dinámica de partícula disipativa (DPD), las técnicas de mecánica cuántica-mecánica molecular (QM-MM), y aproximaciones del efecto solvente como el modelo de apantallamiento como conductor para solventes reales (COSMO-RS). Estas técnicas han demostrado ser muy útiles en la determinación de diversas propiedades como la estructura molecular, y han ayudado a dilucidar la estructura electrónica, reactividad y selectividad de diversos materiales, así como sus propiedades termodinámicas.

En este sentido, la investigación en modelado y simulación en nanociencia en el Instituto Mexicano del Petróleo es cada vez más variada y diversificada debido a que tanto el software como el hardware son cada vez más potentes.

\section{Investigadores especializados en nanotecnología}

El IMP cuenta con recursos humanos altamente especializados y una gran infraestructura para la investigación en nanotecnología; entre el personal altamente calificado existen más de 30 investigadores con grado de doctor y pertenecientes al Sistema Nacional de Investigadores (SNI), además de ingenieros y personal técnico. Si bien el IMP tiene muy diversas áreas de investigación y profesionales en distintas ramas, al menos 15 de nuestros investigadores poseen una vasta experiencia en la preparación, caracterización y aplicación de nanomateriales.

\section{Propiedad intelectual}

El Instituto Mexicano del Petróleo fue creado el 23 de agosto de 1965 como un centro de investigación dedicado al área petrolera. En 1970 le fue otorgada su primera patente, $y$, desde entonces, su acervo se ha incrementado continuamente, consolidándose como uno de los centros de investigación con más patentes registradas anualmente ante el Instituto Mexicano de la Propiedad Industrial. Por ejemplo, solamente en el área de catalizadores, 
cuenta con más de 164 patentes otorgadas, además de 197 en el área de procesos químicos, petroquímicos y refinación, entre muchas otras en las áreas de equipos, aparatos, productos químicos y aditivos. Al mismo tiempo, la investigación científica realizada en el IMP continúa publicándose en prestigiosas revistas científicas internacionales, contribuyendo de manera sustancial al desarrollo tecnológico del país.

La lista completa de patentes se puede consultar en los siguientes vínculos: <http://www.imp.mx/DatosAbiertos/Diccionario.docx>, $<$ http://busca.datos.gob.mx/\#!/conjuntos/patentes/>.

\section{Formación de recursos humanos}

El IMP cuenta con un programa de posgrado donde se pueden obtener los grados académicos de maestro y doctor en ciencias o en ingeniería, así como los diplomas de especialidad en ciencias y especialidad en ingeniería. El posgrado del IMP en su trayectoria histórica de 13 años cuenta con 59 graduados a nivel doctoral y su calidad ha sido avalada por el número de publicaciones con arbitraje internacional con un total de 139 publicaciones en el periodo 2004-2015, así como la productividad de 25 patentes internacionales concedidas y 80 solicitudes, 22 patentes nacionales concedidas y 30 solicitadas, como consecuencia de la importancia de resguardar las investigaciones con carácter de innovación; derivado de esta productividad, actualmente algunos productos se están comercializando por parte del IMP. El $61 \%$ del total de graduados son miembros del Sistema Nacional de Investigadores.

Para el caso de maestría se cuenta con un número de 76 graduados, aunque no es requisito para graduarse la publicación de artículos o solicitud de patente, también se cuenta con productividad científica de 24 derechos de autor y dos patentes. De estos alumnos, el $94 \%$ tuvo beca del CONACyT para realizar sus estudios.

Las áreas temáticas en las que los estudiantes pueden desarrollarse son las de transformación industrial de hidrocarburos o exploración y producción. Entre éstas, las líneas de investigación que podrían relacionarse con la nanotecnología son las de desarrollo de materiales y productos químicos, la de refinación de hidrocarburos, o la de transformación de biomasa, todas ellas en el área de transformación industrial de hidrocarburos.

El programa de posgrado del IMP difunde su oferta académica en el ámbito nacional e internacional. El impacto de esta difusión se puede observar en la diversidad del origen de las solicitudes de ingreso, al momento, de 22 diferentes entidades federativas (Ciudad de México, Edo. de México, Veracruz, Morelos, Tabasco, Hidalgo, Sinaloa, Puebla, Sonora, Guanajuato, San Luis Potos, Tamaulipas, por mencionar algunas). En lo que se refiere al impacto internacional, en la historia del programa de posgrado se han recibido 9 solicitudes de ingreso de diversos países. 
Mundo Nano | Catálogo Nacional | Vol. 9, No. 17, julio-diciembre, 2016 | www.mundonano.unam.mx

FIGURA 5. Equipo robotizado para la preparación de disoluciones, para la síntesis de materiales.

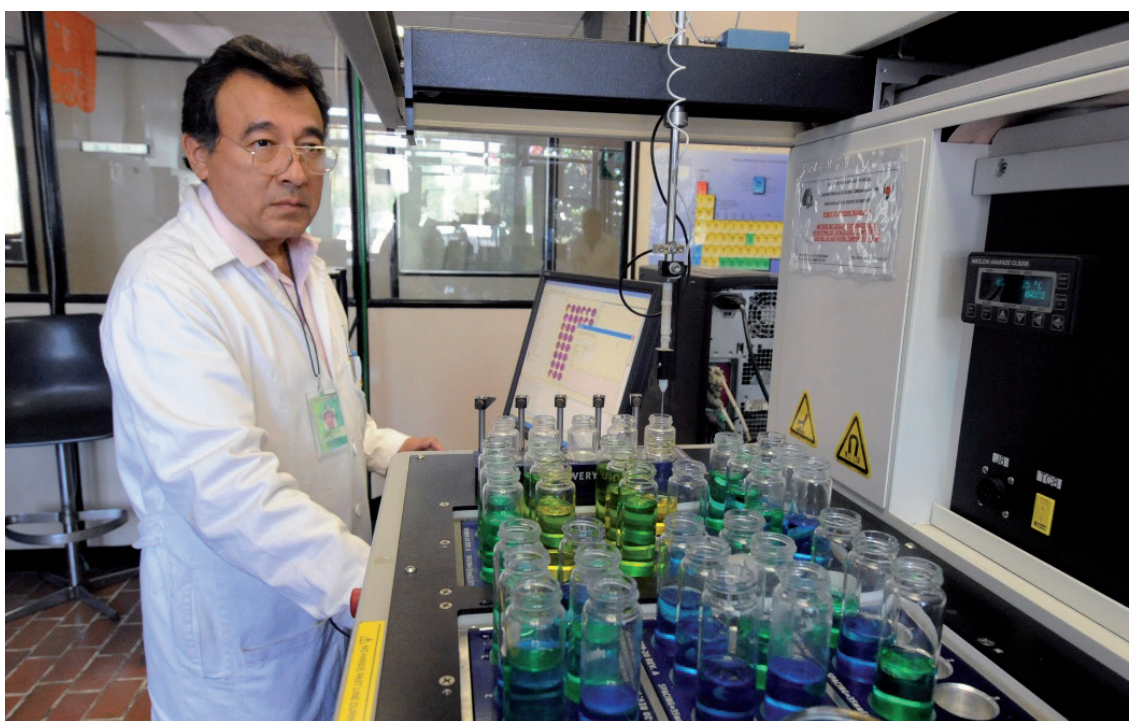

\section{Infraestructura disponible}

El IMP cuenta con gran variedad de tecnología de punta. Por ejemplo, un laboratorio de química combinatoria, equipado con una sección de preparación automática de miligramos de materiales, constituida por dos robots

Figura 6. Equipo de síntesis de materiales por química combinatoria.

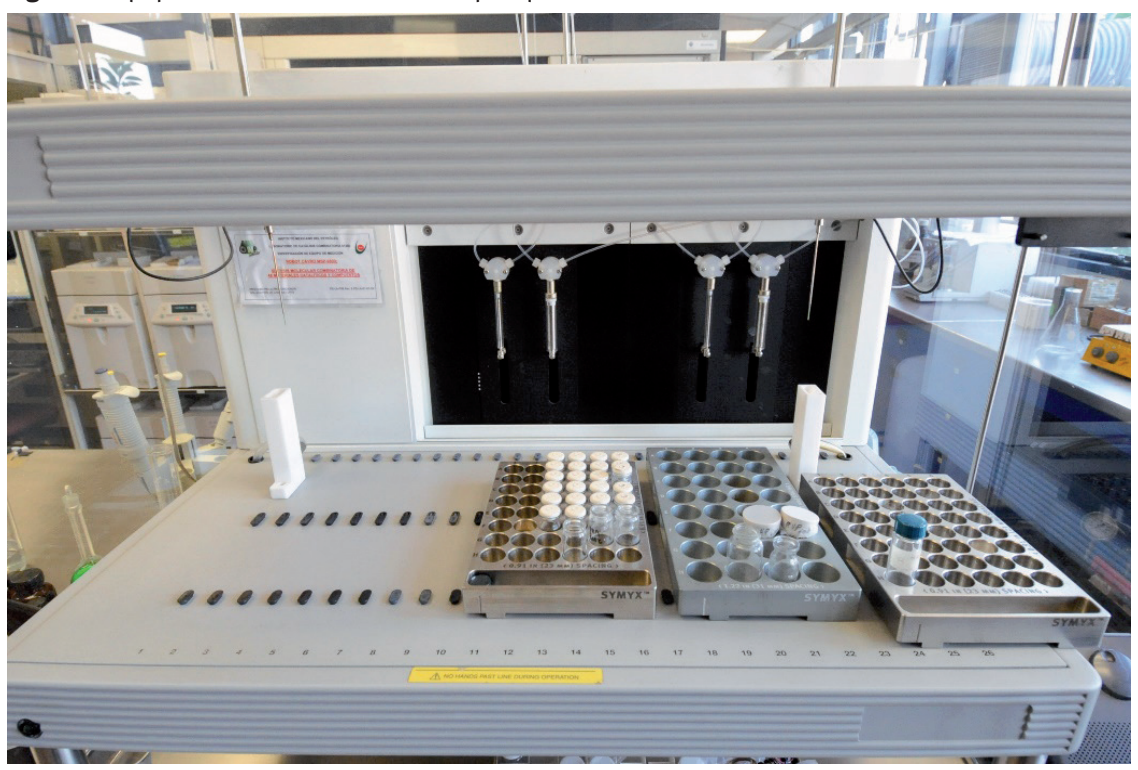


Figura 7. Sección de evaluación de catalizadores del equipo de química combinatoria.

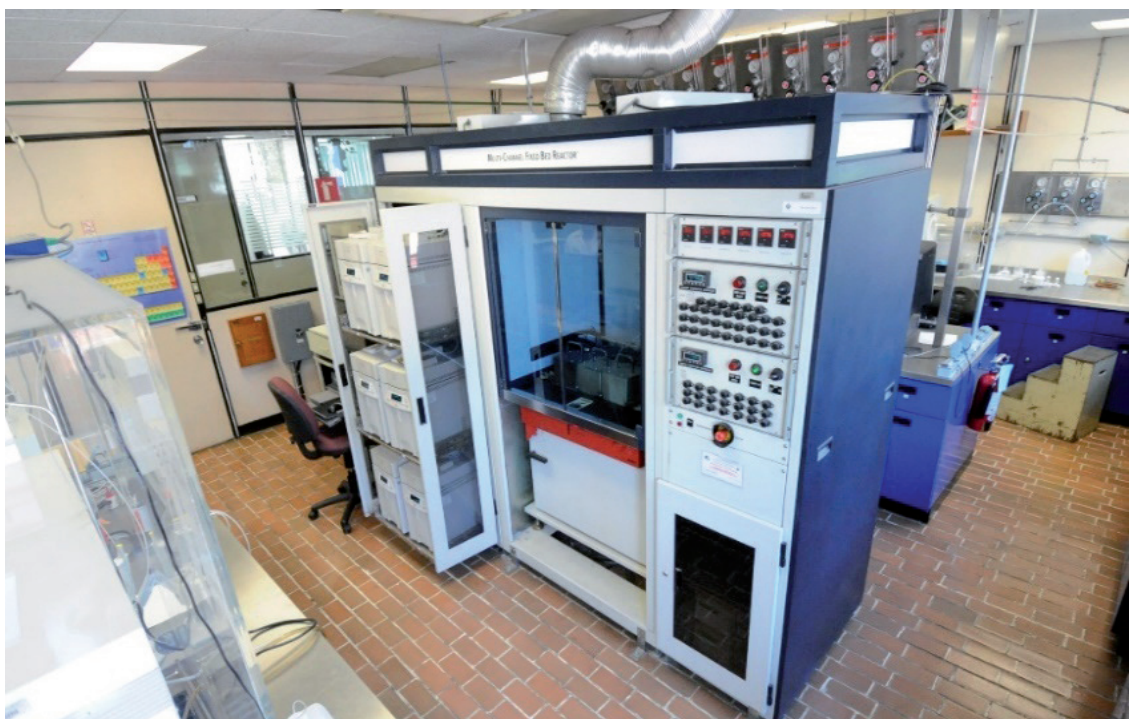

CAVRO, con 48 recipientes de síntesis, bajo atmósfera, temperatura y agitación controladas. Asimismo, el laboratorio tiene una sección de evaluación catalítica de los materiales, empleando moléculas modelo, equipada con 48 microrreactores y un sistema de análisis con 6 cromatógrafos en línea.

Además, el IMP posee diversos laboratorios de síntesis de materiales (adsorbentes, soportes y catalizadores), con infraestructura para preparar desde gramos hasta kilogramos. En los laboratorios de investigación se dispone de infraestructura de material de vidrio y equipo para la síntesis de materiales por diversos métodos: precipitación, coprecipitación, sol-gel, entre otros. Además, los laboratorios están habilitados con trenes de autoclaves, con capacidades desde $50 \mathrm{~mL}$ hasta de $6 \mathrm{~L}$, que operan de forma automatizada para la preparación de materiales mediante métodos hidrotérmicos, tanto a presión autógena como superiores a ésta.

Para el secado de los materiales, se cuenta con equipamiento de estufas atmosféricas y de vacío de diferentes capacidades, así como equipos de secado por aspersión (spray-dryer), para la obtención de lotes desde gramos hasta kilogramos. Para la activación térmica de los materiales, se tienen calcinadores tanto estáticos como dinámicos, habilitados para operar en atmósferas oxidante, reductora o inerte, según se requiera. Se cuenta con calcinadores que pueden operar hasta temperaturas superiores a los $1000{ }^{\circ} \mathrm{C}$, con lotes desde gramos hasta $0.5 \mathrm{~kg}$ de material.

De igual manera, los laboratorios tienen instalada la infraestructura necesaria (amasadores mecánicos, microextrusores y extrusores mecánicos, esferulizador, etc.) para la preparación de materiales y catalizadores másicos o soportados con morfología en extruido (cilíndrico, trilobular, tetralobular, 
FIGURA 8. Equipo de secado por aspersión (spray-dryer), ubicado en el área de microplantas del IMP.

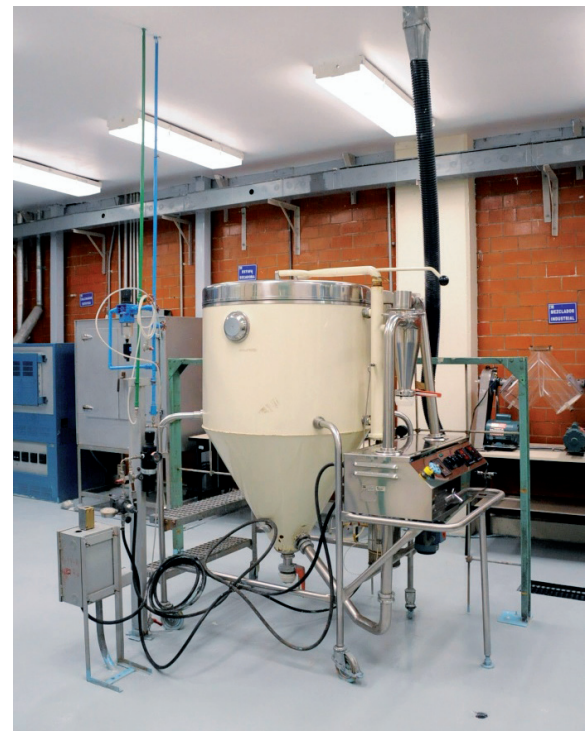

etc., de diferentes diámetros y longitudes), en lotes de gramos hasta kilogramos. Para la preparación de catalizadores con estas morfologías, se cuenta con la experiencia en la formulación, amasado y peptización de pastas de diferentes composiciones.

Existe también una amplia infraestructura para la caracterización de materiales. Para la medición de las propiedades mecánicas de los extruidos fabricados, el IMP dispone de un equipo para la medición de resistencia a la fractura y atrición de catalizadores en forma de extruido ajustándose a los métodos ASTM. Se dispone también de la infraestructura necesaria para la caracterización de los materiales preparados mediante las técnicas de: análisis térmico gravimétrico y térmico diferencial (con análisis mediante espectroscopía de infrarrojo de los productos de descomposición); espectroscopía de absorción atómica; espectroscopía de emisión de plasma; fluorescencia de rayos X; difracción de rayos X; así como fisisorción de nitrógeno para la determinación de las isotermas de adsorción/desorción, el área específica, la distribución de los diámetros de poro, etcétera.

El IMP también tiene equipos de caracterización para la investigación de los materiales a nivel atómico, mediante técnicas especializadas. El Laboratorio de Microscopía Electrónica, el cual es el más completo de México y Latinoamérica. En éste se lleva a cabo la caracterización estructural de nuevos materiales a nivel nanométrico $\left(10^{-9} \mathrm{~m}\right)$, así como el desarrollo de una gran variedad de métodos y análisis. Está conformado por dos microscopios electrónicos de barrido, seis microscopios electrónicos de transmisión, dos microscopios de fuerza atómica, una estación de doble haz (iónico y elec- 
FIGURA 9. Sistema de extrusión mecánica para la obtención de extruidos cilíndricos, tri y tetra-lobulares de distintas longitudes y diámetros.

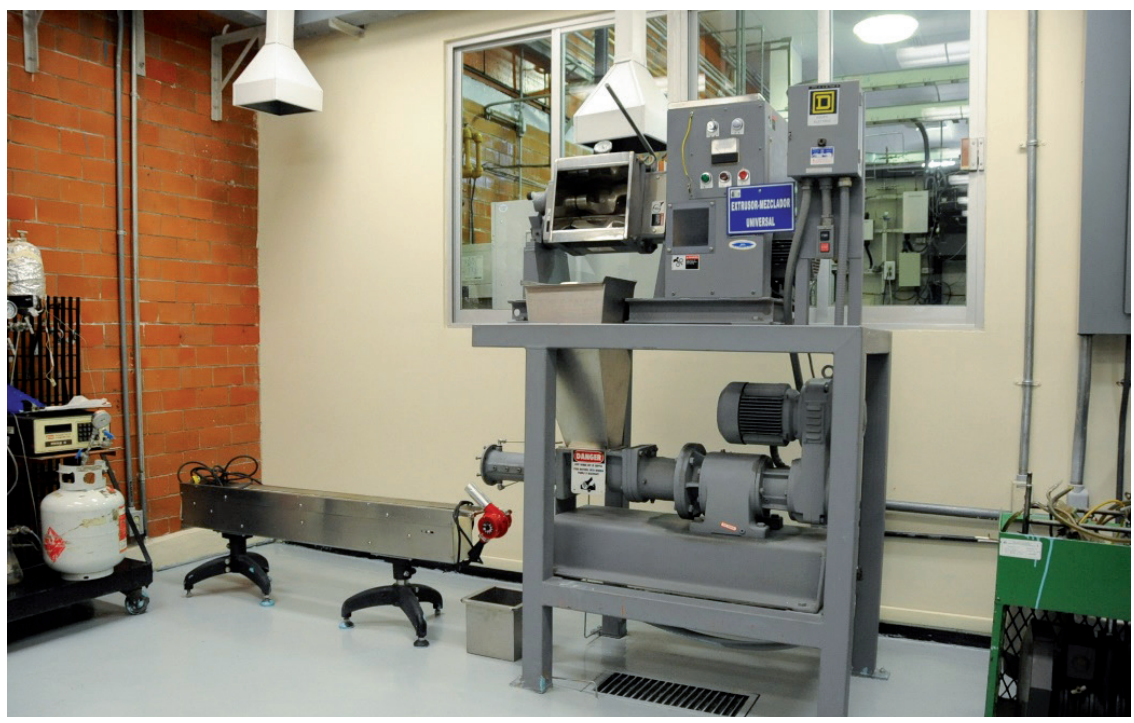

trónico), un nanomanipulador y equipo periférico para la preparación de muestras.

Para su óptima operación, el laboratorio se divide en cuatro grandes áreas:

a) Microscopía electrónica de transmisión, mediante la cual se determina la morfología, tamaño y estructura cristalina de materiales a nivel micrométrico, nanométrico y atómico (hasta $0.85 \AA$ ), para posteriormente correlacionar las propiedades fisicoquímicas, ópticas, magnéticas y electrónicas, con su comportamiento macroscópico.

b) Microscopía electrónica de barrido, en la que se analiza la estructura de la superficie de los materiales (orgánicos, inorgánicos y biológicos) a nivel micro y nanométrico, con el fin de correlacionarla con las propiedades de superficie de la muestra (rugosidad y fenómenos de interface, entre otros). Asimismo, se realizan análisis químicos a escala nanométrica para obtener información de la naturaleza y composición de los materiales.

c) Microscopía de fuerza atómica y tunelamiento, misma que permite estudiar la superficie de las muestras, con alta resolución espacial y capacidad de medición de relieves topográficos y dominios magnéticos. Esta técnica tiene una característica única: su capacidad de manipular átomos y moléculas, modificando local y controladamente la superficie de la muestra para el diseño de nuevos materiales. 
FIGURA 10. Microscopio electrónico de transmisión de alta resolución.

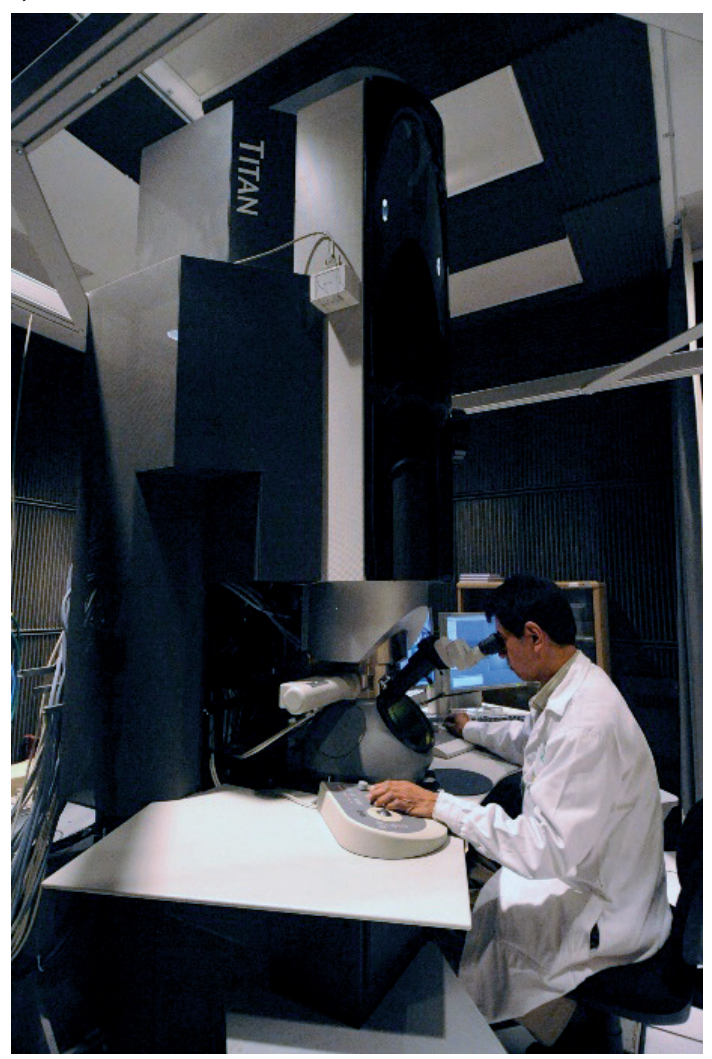

d) Finalmente, la preparación de muestras se efectúa en un área en la que se utilizan varias técnicas, para observar adecuadamente los materiales en los microscopios y hacer cortes transversales con ayuda de un haz iónico, cuya precisión se mide en micras.

En cuanto a la caracterización de la superficie de los materiales, el IMP cuenta con laboratorios de espectroscopía Raman y fotoelectrónica de rayos $\mathrm{X}$ (XPS). El instrumento de espectroscopía Raman tiene un diseño integrado por un triple espectrómetro para una alta estabilidad óptica. La tecnología de filtros holográficos Notch proporciona una muy buena solución para observar números de onda en el espectro visible, hasta $10,000 \mathrm{~cm}^{-1}$. Contempla una tecnología de doble monocromador (modo triple sustractivo) que permite obtener información espectral muy cercana a la línea de láser $\left(5 \mathrm{~cm}^{-1}\right)$. La configuración triple aditiva hace posible realizar un estudio muy preciso de la posición de las bandas Raman, del orden de $0.1 \mathrm{~cm}^{-1}$ facultando la observación de muy pequeñas transiciones de energía. Cuenta con microscopio con confocal acoplado con objetivos de 10X, 50X, y 100X. Tiene una celda Linkam 
FIGURA 11. Microscopía de fuerza atómica (AFM) realizada sobre una película de hidróxidos dobles laminares de composición MgZnGa, depositada sobre ITO.
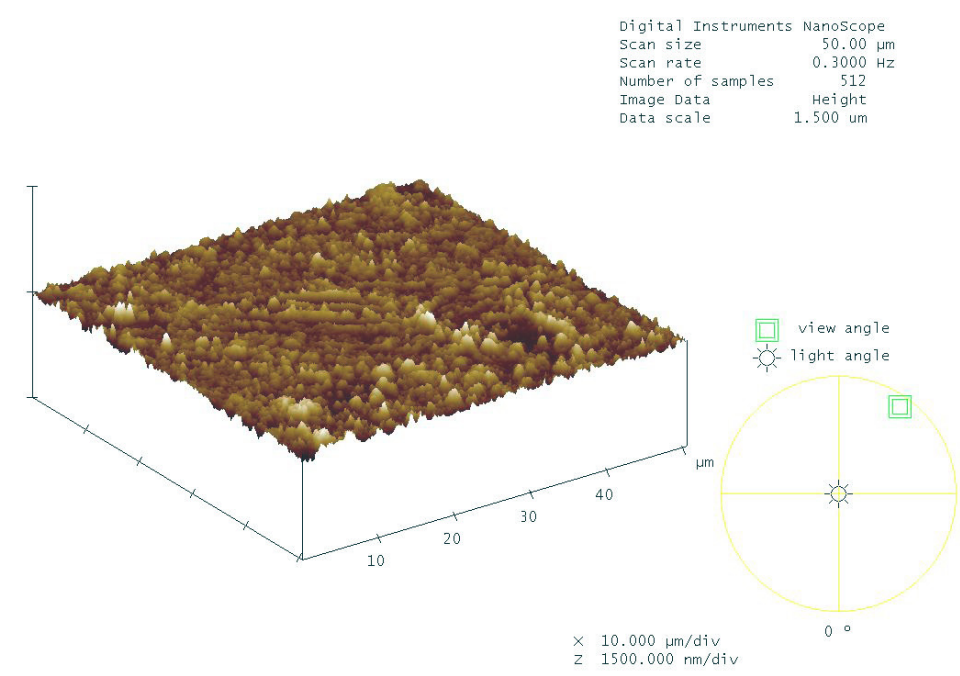

mfa1007-mgznga106c1z1.000

THM600 de calentamiento/enfriamiento $\left(-196{ }^{\circ} \mathrm{C}\right.$ a $\left.600{ }^{\circ} \mathrm{C}\right)$ donde es posible hacer estudios de transformaciones de fases cristalinas y modificaciones superficiales, por efectos de temperatura o atmósfera oxidante o reductora y estudiar además interacciones gas-sólido bajo atmósfera controlada. También se cuenta con otra celda Linkam CCR1000, la cual es una celda de reacción para estudiar transformaciones estructurales durante un ambiente reductivo u oxidativo o inclusive seguir una reacción catalítica acoplando un analizador cromatográfico a la salida.

Esta técnica de análisis se hace necesaria para poder comprender los mecanismos de reacción que participan en muchos procesos importantes. Esto facilita el diseño y desarrollo de nuevos materiales y su aplicación de manera óptima. Dado que fortalece el conocimiento de caracterización in situ e in operando, permite adquirir información de tipo estructural de catalizadores y su fase activa, cerca de las condiciones reales de reacción dentro de un proceso catalítico.

La técnica de espectroscopía Raman es de carácter no destructivo. Las muestras a analizar no requieren ningún tratamiento previo, su naturaleza puede ser sólida, con distintos estados de agregación como polvos o piezas; líquida o gaseosa, utilizando celdas adecuadas para ellas. Dado que se trata de una técnica de microespectroscopía, el tamaño de la muestra puede ser microscópico o analizar de forma diferenciada zonas distintas dentro de la misma.

En el IMP hay también un laboratorio de espectroscopía fotoelectrónica de rayos $\mathrm{X}$ (XPS). El XPS es una técnica de análisis de superficies, eficaz y 
FIGURA 12. Equipo de espectroscopía Raman (Yvon Jobin Horiba, T64000) equipado con doble monocromador (modo triple sustractivo)

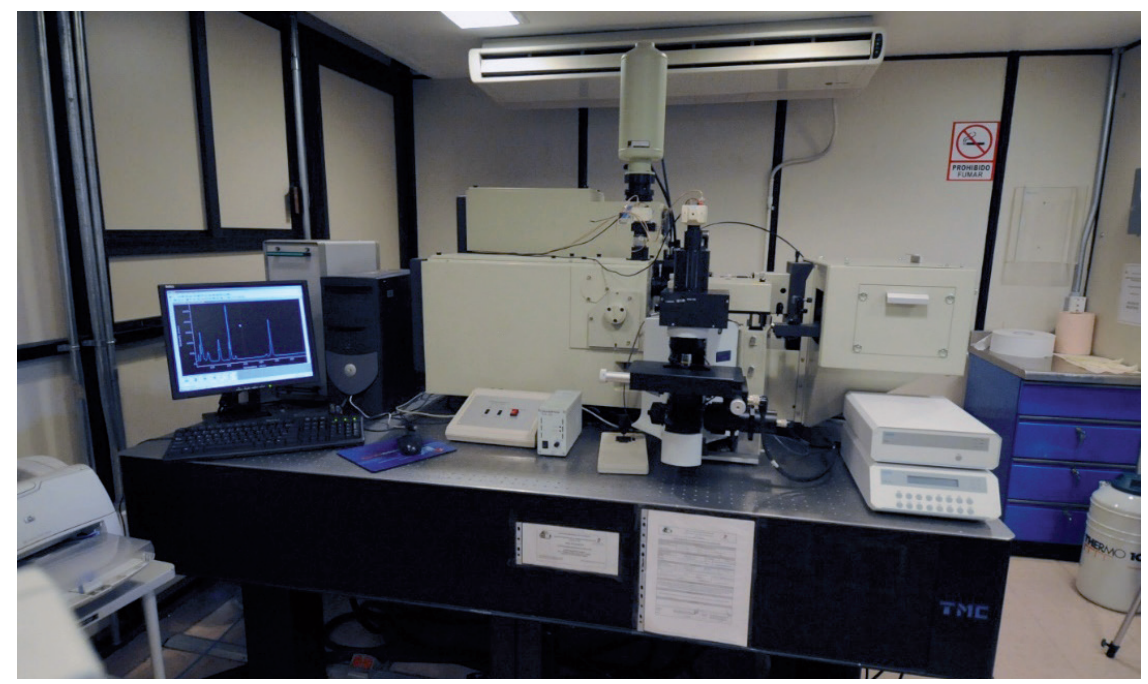

ampliamente utilizada. Una muestra es irradiada por baja energía de rayos $\mathrm{X}$ en ultra alto vacío. Esto causa una fotoionización de átomos en la superficie de la muestra: los fotoelectrones son emitidos desde niveles de energía determinados por la estructura electrónica del espécimen. La técnica del análisis de XPS examina las energías cinéticas de estos fotoelectrones para determinar su distribución de energía. De los resultados de este análisis es posible la identificación de los elementos presentes, sus estados químicos y la cuantificación de ellos.

Por otra parte, existen tres equipos de espectroscopía de infrarrojo por transformada de Fourier (FTIR): un espectrómetro FTIR NICOLET, modelo 710, un espectrómetro FTIR NICOLET, modelo MAGNA-560 y un espectrómetro FTIR NICOLET, modelo PROTEGE 460. Durante los procesos de desarrollo de materiales con propiedades especiales y con capacidad para ser utilizados como soportes de catalizadores, catalizadores o bien en procesos fotocatalíticos, es necesario llevar a cabo la caracterización de los mismos.

La espectroscopía FTIR ha sido una de las técnicas espectroscópicas pilares en el desarrollo de nuevos materiales y catalizadores vía la caracterización estructural, estudios por marcado isotópico y la determinación de las propiedades fisicoquímicas por la adsorción-desorción de moléculas sonda, tanto a alta como a baja temperatura. En particular, dentro de las más utilizadas podemos mencionar la adsorción-desorción de piridina y dióxido de carbono, las cuales permiten examinar las propiedades ácido-base de los materiales, así como del monóxido de carbono a baja temperatura (100K), que posibilita determinar sitios activos con deficiencias electrónicas. Actual- 
FIGURA 13. Equipo de espectroscopía fotoelectrónica de rayos X (XPS) THERMO-VG SCALAB 250 equipado con fuente de rayos $\mathrm{X}, \mathrm{AlK} \alpha \mathrm{X}(1486.6 \mathrm{eV})$ y analizador hemisférico.

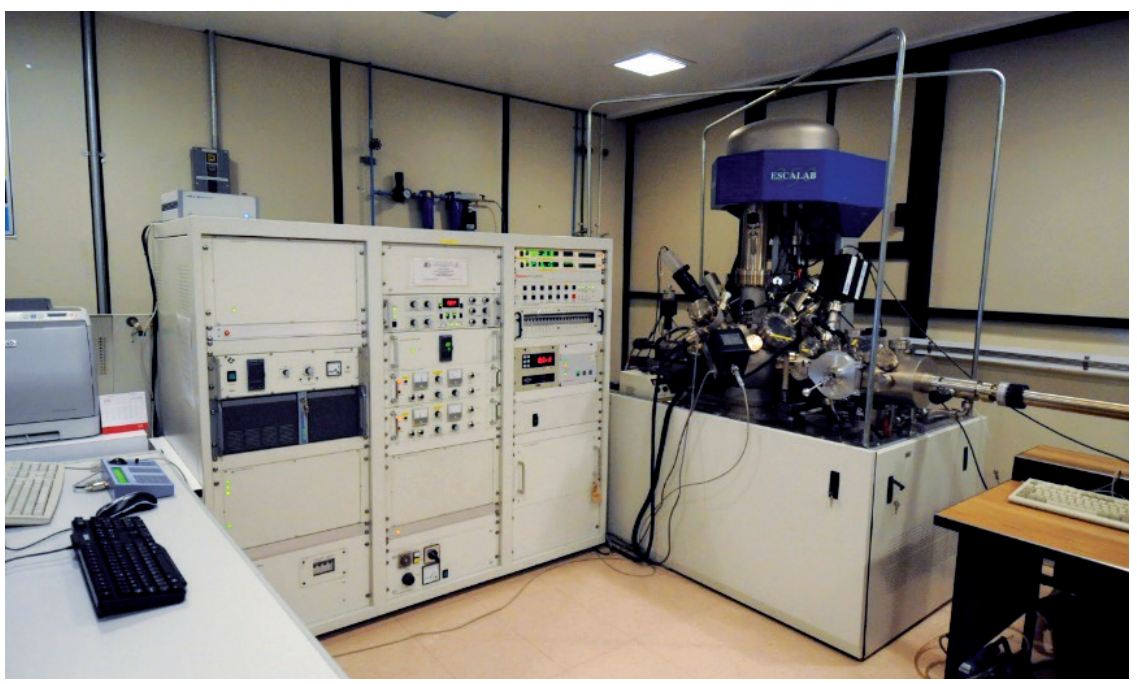

mente, se cuenta con equipos FTIR que permiten aplicar técnicas novedosas de caracterización en el campo de la catálisis y los materiales, como son los sistemas de caracterización en el modo OPERANDO hoy en día en boga y las técnicas de STEP-SCAN que facilitan seguir procesos efectuados a alta velocidad y capaz, además, de trabajar en todo el rango espectroscópico, aún desde el ultravioleta-visible (UV-Vis).

Posteriormente, el análisis de los fenómenos de transición por excitaciones electrónicas y los procesos de relajación son importantes para determinar las propiedades de estos materiales así como las energías fotónicas necesarias para superar la energía de banda prohibida entre la banda de conducción y la banda de valencia. Por otro lado, los nanomateriales presentan efectos electrónicos propios de las geometrías que estas nanoestructuras adoptan, por efecto de su tamaño, mismas que pueden ser estudiadas utilizando la técnica de resonancia plasmónica en el ultravioleta-visible (UV-Vis).

En el laboratorio de resonancia magnética nuclear (RMN) se realizan análisis que proporcionan datos en relación con el entorno de la estructura en un material, principalmente de los estados de coordinación o corrimientos isoméricos, análisis estructural y comportamiento del átomo en todo el sólido. La RMN de líquidos se utiliza como una poderosa técnica analítica cuya aplicación es amplia. En general esta técnica permite la elucidación de estructuras moleculares, el estudio de polímeros y la determinación cuantitativa de mezclas; asimismo, posibilita determinar parámetros moleculares promedio, tales como el factor de aromaticidad, el número de sustituyentes alquilo en anillos aromáticos y la distribución de tipos de 
Figura 14. Equipo de resonancia magnética nuclear de sólidos (RMN).

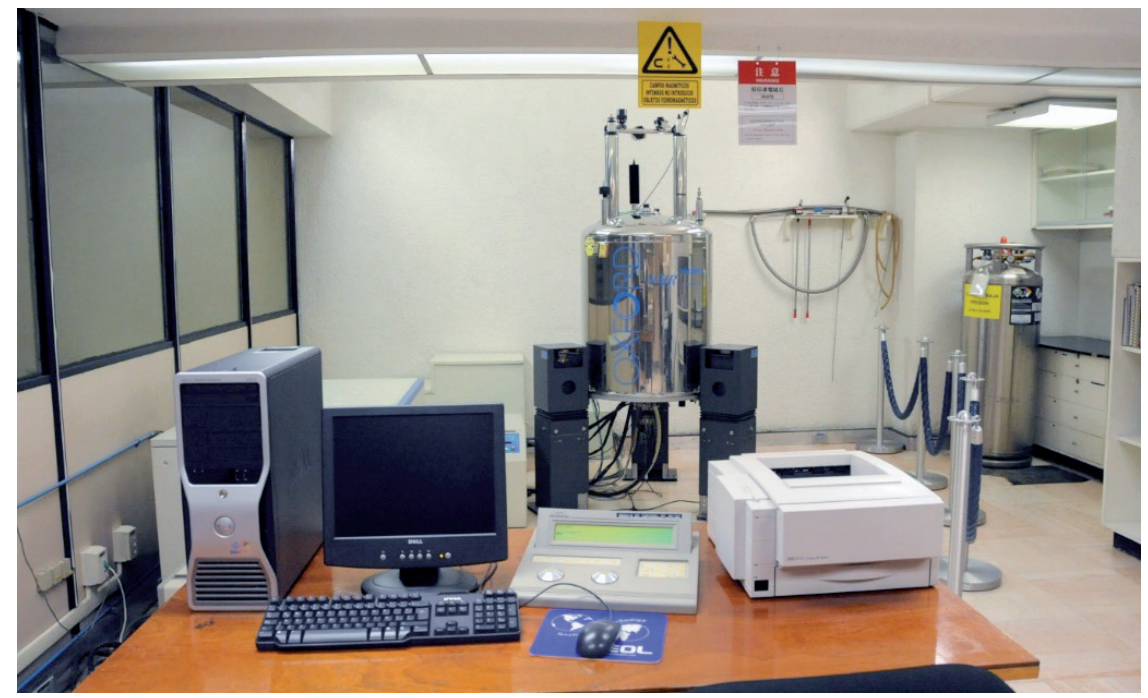

carbono en mezclas complejas de hidrocarburos. Entre las aplicaciones de la RMN de sólidos en el IMP, se encuentran las de el estudio de materiales zeolíticos, en donde se han identificado los tipos de aluminio presentes, la relación $\mathrm{Si} / \mathrm{Al}$, etc.

La aplicación de la RMN de sólidos en catálisis heterogénea provee información esencial para avanzar en el diseño de nuevos catalizadores activos y selectivos para su aplicación en diferentes procesos químicos. Por sus características, esta técnica permite investigar aspectos diferentes de un sistema catalítico: i) caracterización estructural de los catalizadores; ii) interacción del catalizador con moléculas sonda y reactivos; iii) estudio de mecanismos e intermediarios de reacciones químicas. Desde el desarrollo inicial de la RMN de sólidos, las zeolitas (silicatos y aluminosilicatos) y los zeotipos (alumino-fosfatos y silico-alumino-fosfatos) han ejercido una gran atracción sobre los investigadores por sus únicas propiedades estructurales y químicas. Además del interés académico, estos materiales tienen aplicaciones industriales potenciales, como catalizadores en procesos petroquímicos, así como adsorbentes e intercambiadores iónicos. La RMN de $27 \mathrm{Al}$ y 29Si (31P en aluminofosfatos) da información del entorno local de los átomos en la red, que complementa los datos de difracción de rayos $\mathrm{X}$ y otras técnicas, en la elucidación estructural de los materiales. La RMN de $1 \mathrm{H}$ y su interacción con moléculas sonda permite estudiar las propiedades ácidas.

Respecto a la espectroscopía Mössbauer, para la cual también se cuenta con un laboratorio, ésta tiene numerosas aplicaciones en el campo de la corrosión, debido a su sensibilidad para el estudio de materiales sólidos que contienen hierro, así como los productos de corrosión de ductos que 
FIGURA 15. Sistema de evaluación catalítica con reactor en flujo continuo y lecho fijo, a nivel microrreacción, para el hidrotratamiento de destilados intermedios de refinerías.

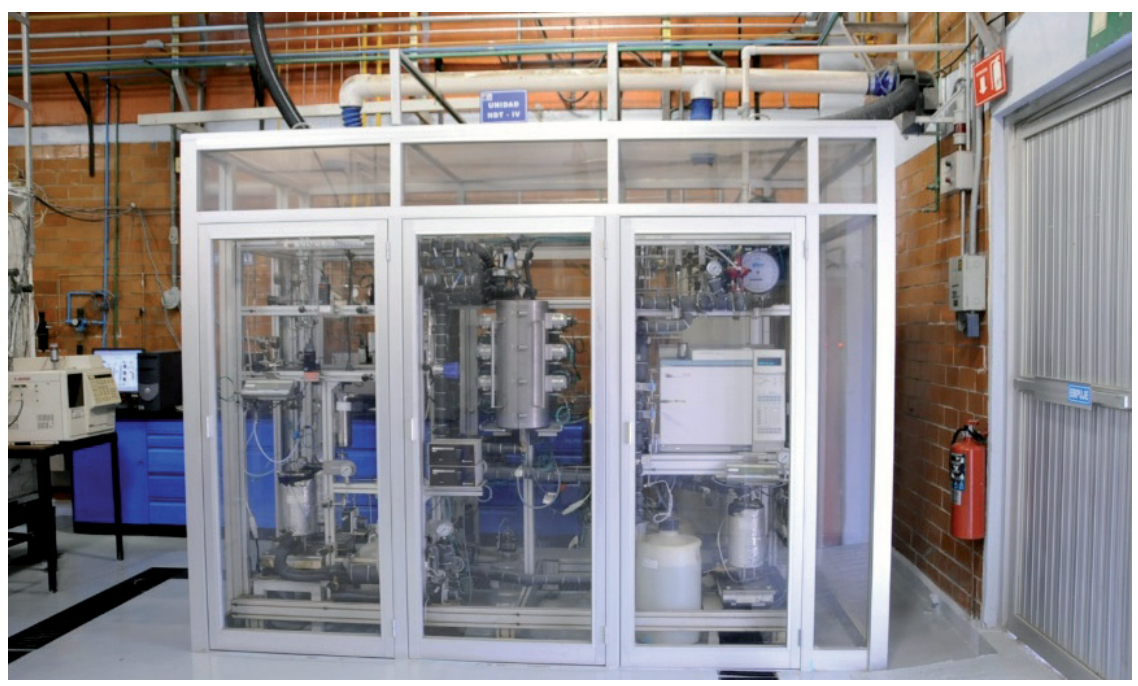

transportan crudo, gasolina y combustóleo ( $\mathrm{FeS}, \mathrm{FeC}$ y FeO). Su sensibilidad y alcance facilitan la revelación de aspectos no expuestos por otras técnicas. Se pueden determinar compuestos de hierro cuyos porcentajes son mayores que $0.5 \%$ en peso. Estos estudios se complementan con análisis de fases por difracción de rayos X (DRX) y análisis semicuantitativo por fluorescencia de rayos $\mathrm{X}$ (FRX). La identificación se realiza por medio de las propiedades magnéticas y eléctricas de los componentes de hierro.

Por otra parte, en el IMP hay también diversos laboratorios para evaluación catalítica, se cuenta con infraestructura de reactores por lotes para la evaluación de prototipos catalíticos con moléculas modelo y cargas reales. En el laboratorio de microrreacción se lleva a cabo la evaluación continua de catalizadores con cargas sintéticas o reales de refinería. Además, existen laboratorios de plantas piloto, los cuales están habilitados con equipos a escala micro y semicomercial que permiten simular la operación de las unidades industriales con una menor inversión, reduciendo gastos de operación y de insumos de materias primas, catalizadores, energéticos y servicios auxiliares.

Como se ha referido, el IMP tiene la capacidad para efectuar el escalamiento de materiales catalíticos, realizar la evaluación de procesos en microplantas y de materiales en microsistemas. Para ello se dispone de reactores de lecho fijo, ebullente y lecho fluidizado transportado; así como de reactores que trabajan en régimen de tanque agitado, isotérmico y adiabático, y equipos de destilación. La operación de estas unidades es continua, automatizada y con la capacidad de obtener registros electrónicos; además se cuenta con soporte analítico según las necesidades del usuario. 
Mundo Nano | Catálogo Nacional | vol. 9, No. 17, julio-diciembre, 2016 | www.mundonano.unam.mx

FIGURA 16. Planta piloto de evaluación de catalizadores en el proceso de desintegración catalítica (FCC) con gasóleos de refinerías.

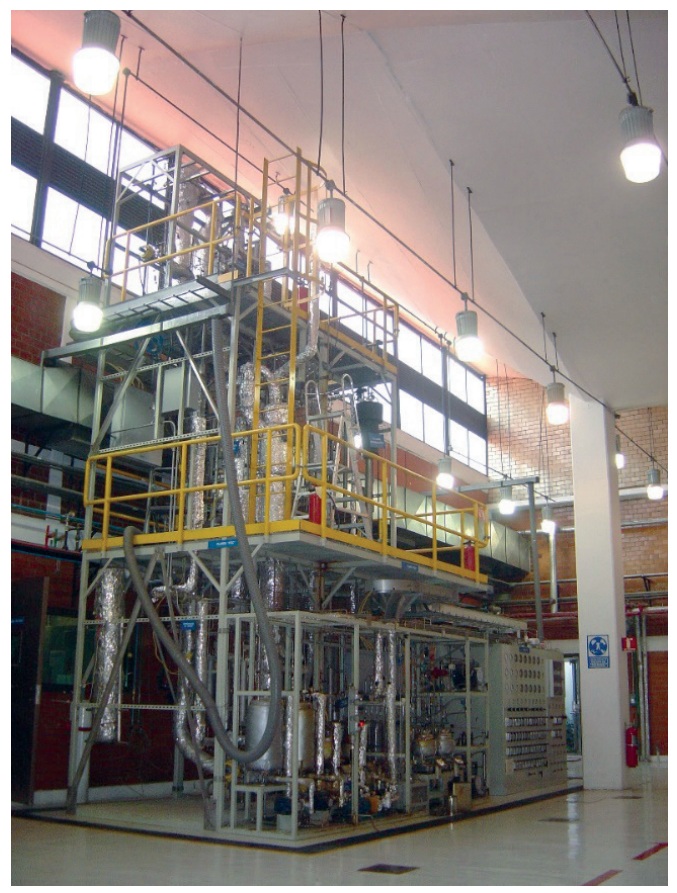

El laboratorio de plantas piloto y escalamiento de catalizadores da apoyo experimental a los proyectos de investigación, desarrollo tecnológico y de servicios, relacionados con los procesos de refinación y petroquímica.

\section{Sitios de interés}

- $\quad\langle$ http://www.imp.mx>

- <http://busca.datos.gob.mx/\#!/conjuntos/revistas-arbitradas/> 